\title{
On-line Monitoring of Temperature in Power Transformers using Optimal Linear Combination of ANNs
}

\author{
M. K. Pradhan \\ T. S. Ramu \\ Department of High Voltage Engineering, \\ Indian Institute of Science, Bangalore (INDIA), E-mail: manoj@hve.iisc.ernet.in.
}

\begin{abstract}
Inordinate temperature rise in a power transformer due to load current is known to be the most important factor in causing rapid degradation of its insulation and decides the optimum load catering ability or the loadability of a transformer. The Top Oil Temperature (TOT) and Hottest Spot Temperature (HST) being natural outcome of this process, an accurate estimation of these parameters is of particular importance. IEEE/ IEC among others, have proposed procedure to estimate the temperatures, however, the accuracy of the predictions are not always as good as are desired. Unacceptable temperature rise may occur due to several fault conditions other than overloading, and hence warrant an online monitoring of the transformer. This paper presents an improved model for predicting TOT and HST based on Artificial Neural Network (ANN). A series of network architecture (p-trained network) have been proposed and trained for working out this task and are further optimally combined to give an improved accuracy.
\end{abstract}

Keywords-Hot Spot Temperature, Top Oil Temperature, ANN, Optimal Combination, Prediction Accuracy

\section{INTRODUCTION}

$\mathrm{T}$ RANSFORMERS are vital components in a power system and a fault in this link causes considerable loss of revenue to the utility, besides adversely affecting the system reliability. Localized temperature rise in the winding takes place due to load current coupled with short time over-loads, which the system is asked to cater to. Hot Spots, regions of inordinate temperature rise in the winding and top surface of insulating oil (Hottest spot temperature, HST, and Top Oil Temperature, TOT) causes rapid thermal degradation of insulation and subsequent thermal run away. In order to avoid catastrophic failure of such expensive equipment, monitoring of the condition of insulation there- in is undertaken on a regular basis. The genesis of the two zonal temperatures, TOT and HST, is a complex process in itself, vitiated further by the thermal inhomogeniety of the winding [1] and dynamic nature of the load and ambient [2].

The thermal conductivity of the insulation part being largely different from that of the conductor and the fact that there is an anisotropy in this quantity in both radial as well as axial directions complicates the estimation procedure. In the recent past, technical bodies, IEEE and IEC, have suggested empirical methods for estimating the temperature extremes. In view of the fact that the estimation of temperature using the formulations there in is not as accurate as are desired, new and improved methods need to be developed to mitigate the situation.

A perusal of current literature in this area indicates that different empirical models, viz. IEEE and IEC [3], have been proposed for the estimation TOT. Lesieutre et al. [2], [4], have suggested a modification to incorporate dynamic ambient. It is seen that the error in the estimation of TOT in such calculations is unacceptably high, so that no meaningful conclusions can be drawn there-from.

Estimation of constants of the empirical models [2]-[4] by a linear regression procedure is data driven and generalizations are not easily possible. Also, a large database is always required to make the estimates less variant, as these models are not robust or adaptable in a stochastic sense. A research group at Arizona, USA, [5], has proposed a methodology based on RNN to predict TOT in power transformers. Although, recurrent networks can some times outperform static networks, for temporal problem, they appear more difficult to train optimally. Earlier experiments indicated that the parameters thereof suffer irreconcilable entanglement in excising minima in sub optimal solutions.

Feed- forward neural network (FFNN) or Multi-Layer Perceptron (MLP) models, have been used in several engineering applications, such as, load forecasting, non-linear control, system identification, pattern recognition and the like. It is intended to use this technique here with certain subtle modifications as this model has capability of capturing any non-linear relation between input parameters and output of a complex physical process. Numerous training algorithms for parameter optimization of MLP, suitable for this particular application, already exist and opportunity has been taken of this precedence here to try and suggest a more effective model.

Further, temporal aspects can easily be built into the network by the use of memory. The approach for building non-linear dynamical system is straightforward; while static FFN accounts for non-linearity, the memory accounts for time [6].

The authors have tried to incorporate both TOT and HST in one framework of reference and developed an integrated model suitable for online monitoring. Optimal selection of network topology for the problem has not been taken rigorously and the present work aims at suggesting an alternate NN topology which uses regularization techniques, called Bayesian regularization (BR) [7] to improve the generalization performance the network. In an effort to improve the accuracy of estimates, a number of networks are trained for the same task and combined using the principle of optimal linear combination (OLC) of neural networks [8]. The method is demonstrated using the data given in literature [3].

To provide guidance on risk associated with higher operating temperature in an actual situation, four standard loading cycles have been defined [3]. For each temperature, a certain marked risk is involved. The Loading cycles can one be or a combination of the following;

- Normal Loading (NL)

- Planned Load beyond Nameplate Ratings (PLBN)

- Long-term Emergency (LTE) loading

- Short-term Emergency (STE) loading

For the sake of simplicity, it will be assumed that at any given point of time, only one condition described above prevails. 


\section{Prediction of Temperature uSING ANN}

An artificial neural network consists of a topology and a set of rules that govern the static and dynamic aspects of network in question. The network topology or architecture represents the way in which 'neurons' are connected to form the network Topology of a neural network plays a pivotal role in its function and performance. Feed forward neural networks have been used for enumerating the approximations generic to the problem. In the following sections, two relevant network architectures [6] have been discussed in some detail.

Multi-Layer Perceptron is a feed forward network with one or more hidden layers of neurons, is an elementary neural network paradigm, and have been successfully applied to solve involved and diverse problems by training them in supervised manner. The distinctive features of this network are; non-linear activation function in the hidden layer neuron and the network's ability to accommodate non-linearity of any complex process as also is endowed with a high degree of connectivity. This feed forward network is trained by a conventional back-propagation (BP) algorithm.

While a Multi-layer Perceptron can be trained to take account of non-linear relation existing between an input and an output parameter of a complex process, it suffers from a serious drawback in that it fails to represent temporal evolution of input pattem, if present. A temporal processing unit, in the neural network is composed of a tapped delay line associated memory, (TDM) with taps connected to the synapses of a neuron. The TDM captures temporal information contained in the input signal and the neuron embeds that information in its synaptic weights. The integration of MLP and tapped delay line memory give rise to a model called focused lagged feed forward or a Temporal Neural Network (TNN). The generalized dynamic equation governing input output relations of a TNP is given here under.

$$
y(n)=\psi\left(x_{1}(n), \ldots, x_{1}(n-d), x_{2}(n), \ldots \ldots, x_{2}(n-d)\right)
$$

In developing the NN model, there is a need for a prior knowledge of the $\mathrm{I} / \mathrm{O}$ pattern, ex. the size of the lag variables. There seem to be no information on this aspect in the literature. Since the thermal time constants of the different components in a transformer is generally known, it is possible choose the delay term, $\mathrm{d}$, ' such that the equation $(d \times \Delta t>\tau)$ is fully defined. In this equation, $\Delta t$ is the quantization time step or data sampling time and $\tau$ is the thermal time constant. The temperature at $i_{t h}$ instant of time is a function of load, ambient temperature at that instant and their corresponding lags in the computation of TOT. Estimation of HST, requires, besides these two variables, the TOT also. Neural network training can be made more efficient if the data is pre-processed by transforming $\mathrm{I} / \mathrm{O}$ variables on to a different appropriate domain. There are several techniques for preprocessing, e.g; scaling the all the parameters over a domain $[0,1]$ or $[-1,1]$. An equally effective method of scaling the inputs and targets is to nornalize them to an appropriate with zero mean and a standard deviation of unity. In some situation the dimension of the input vector is very large, but the components of the vectors are highly correlated (redundant). It is useful in such situations to prune the dimension of the input vectors. An ef- fective procedure for doing this task is the principal component analysis (PCA).

\section{IMPROVING GENERALIZATION}

Modeling based on ANN requires a careful selection of network topology and an appropriate learning algorithm to avoid complications arising out of possible existence of 'local minima'. This facilitates achieving an acceptable generalization performance and hence a good prediction accuracy. The algorithm, which consistently produces networks with minimal generalization error, is referred to as regularization. A network of a reasonable size should be able represent, sufficiently accurately, the actual temperature rise mechanism. It is difficult to guess which training algorithm will satisfy above condition given that appropriate topology is selected. In the following sections, an improved, automated regularization procedure called the Bayesian regularization, has been discussed in some detail.

\section{A. Automated Bayesian Regularization}

The feed-forward NN (MLP/TNN) are trained using supervised learning with a training set of inputs and target in the form, $\left\{p_{1}, t_{1}\right\},\left\{p_{2}, t_{2}\right\},\left\{p_{3}, t_{3}\right\}, \ldots .\left\{p_{N_{1}}, t_{N_{1}}\right\}$. The objective of this training program is to minimize the sum square errors:

$$
E_{D}=\sum_{i=1}^{N_{1}}\left(t_{i}-a_{i}\right)^{2}
$$

Where, $a_{i}$ is the response of the NN to a input pattern. Such a training produces a network wherein small, known amounts of errors are imparted to the training set and at the same time, the network is coaxed to respond in the right sense to the new inputs (testing set). When the network satisfies this condition, it is means that the generalization is satisfactory. The process function is constrained to conform to a high degree of smoothness by assigning the appropriate weights to the network configuration. A further step in the regularization adds one more additional term to objective function $F=F_{D}$, so that;

$$
F=\lambda E_{D}+\eta E_{W}
$$

Where, $E_{W}$ is the sum of squared network weights, $\eta$ and $\lambda$ are objective function parameters. The relative size of $\eta$ and $\lambda$ dictates the emphasis for training. If $\eta<<\lambda$, training algorithm drive the errors smaller, If $\eta \gg>\lambda$, training will emphasize weight size reduction at the expanse of network errors, thus producing smoother network response. Hence, the main problem of implementing regularization appropriate is to set correct values to objective function parameters. It involves optimization of objective function parameters, $\eta$ and $\lambda$ in an Bayesian framework. The Bayesian optimization of regularization parameters requires the computation of Hessian matrix of objective function $H$ [7]. A Gauss-Newton approximation to computation of Hessian matrix is includeded in the Levenberg-Marquardt optimization algorithm to locate the error minima [6]. It can be shown that, $\hat{\eta}, \hat{\lambda}$, the optimized value of the objective function parameters can be put in the following form;

$$
\hat{\eta}=\frac{\gamma}{2 E_{W}(\hat{\mathbf{w}})} ; \hat{\lambda}=\frac{N_{1}-\gamma}{2 E_{D}(\hat{\mathbf{w}})} ; \gamma=K-2 \hat{\eta} \operatorname{tr}(\hat{\mathbf{H}})^{-1}
$$


Where, $\gamma$ is called the effective number of parameters and $K$ is available number of parameters. An intutive guess can be made as to number of neurons in the hidden layer, then the effective number of parameters, $\gamma$, can be calculated. If $K \approx \gamma$ then number of neurons is to be increased, if $K>\gamma$, means the network is large enough to handle the complexity of the problem space. It can be seen from Table I that the desired number of parameters, $\gamma$ seems to be independent of selected number of parameters, $K$.

TABLE I

BAYESIAN REGLLARIZATION

\begin{tabular}{|c|c|c|c|c|}
\hline \hline NN & Topology & No. of & \multicolumn{2}{|c|}{ Parameters } \\
\cline { 4 - 5 } & selected & Epoch & used $(\gamma)$ & available $(K)$ \\
\hline NN1 & $14-12-1$ & 175 & 123 & 161 \\
NN2 & $14-04-1$ & 175 & 124 & 241 \\
NN3 & $14-10-1$ & 175 & 132 & 193 \\
NN4 & $14-20-1$ & 175 & 128 & 241 \\
\hline
\end{tabular}

\section{B. Optimal Linear Combination of Networks (OLCN)}

The Neural Network based modeling often requires trying multiple networks with different topology and training parameters, but, typically, only one of the trained networks is selected as best and rest are discarded. As has already been explained a linear combination of the the corresponding outputs of a screened set of trained NNs give better generalization performance than a single best NN [8]. A schematic diagram of the model has been shown in Fig.1.

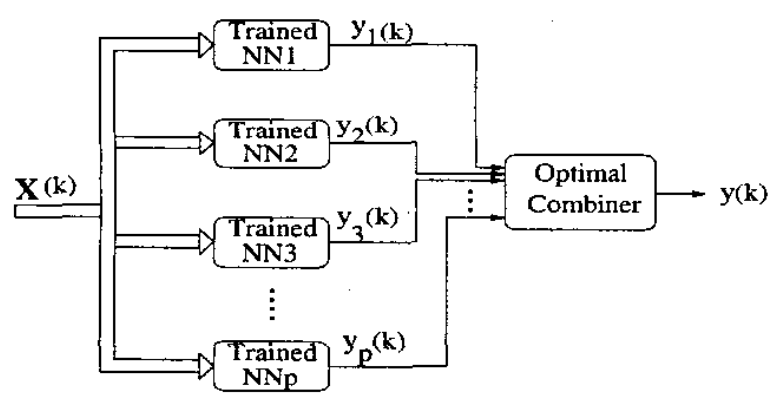

Fig. 1. Represenation of combining the p-trained networks

A trained NN accept a vector valued input $x$ and returns the scalar output (response) $y(x)$. The approximation error in this case is $\delta(x)=t(x)-y(x)$. Where $t(x)$ is the desired response to input $x$. A linear combination of the outputs of the $p$ trained network returns, output and corresponding error as in Eq. (5);

$$
\bar{y}(\vec{X} ; \vec{\alpha})=\sum_{j=1}^{p} \alpha_{j} y_{j}(x) ; \bar{\delta}(\vec{X} ; \vec{\alpha})=t(x)-\bar{y}(\vec{X} ; \vec{\alpha})
$$

Where, $y_{j}(x)$ is the out put of $j_{t h}$ network, $\vec{\alpha}_{j}$, is the weight associated with $y_{j}(x) ; j=1,2, \ldots ., p$.

The OLC is defined by optimal combination-weights vector, $\hat{\alpha}=\left(\alpha_{1}^{*}, \alpha_{2}^{*}, \ldots, \alpha_{p}^{*}\right)$ that minimizes the expected loss;

$$
\int_{S} \ell(\bar{\delta}(X ; \vec{\alpha})) d F_{\vec{X}}
$$

Where $S$ is the support of distribution function $\left(F_{\vec{X}}\right)$ and $\ell$ is a loss-function. In this case squared error loss function, $\ell(\bar{\delta})=\bar{\delta}^{2}$ has been taken.

\section{B.1 Unconstrained Method $\left(M_{1}\right)$}

If the condtion in equation (6) is imposed to determine OLC parameters, then it is called unconstrained OLC weights for gaussian distribution of data set can be calculated as follows;

$$
\hat{\alpha}=A^{-1} * B
$$

Where, $\mathrm{A}$ is a $p \times p$ matrix and $\mathrm{B}$ is a $p \times 1$ vector, can be evaluated as;

$a_{i j}=\frac{\sum_{k=1}^{|N|} y_{i}\left(x_{k}\right) * y_{j}\left(x_{k}\right)}{|N|} ; \quad b_{i}=\frac{\sum_{k=1}^{|N|} t\left(x_{k}\right) * y_{i}\left(x_{k}\right)}{|N|} \forall i, j$

\section{B. 2 Constrained Method $\left(M_{2}\right)$}

If the parameters, $\alpha_{j}$ further constrained such that it satisfies equation (6) and (8)

$$
\sum_{j=1}^{p} \alpha_{j}=1
$$

Then, $\alpha_{j}$ are called 'constrained OLC weights' and following are the formulation for computating constrained weights for data from normal distribution.

$$
\hat{\alpha}=\frac{C^{-1} * 1^{t}}{1^{t} * C^{-1} * 1}
$$

Where $C$ is $p \times p$ matrix and $\mathbf{1}^{t}$ is a $p \times 1$ vector with all its components equal to one. The components of $C$ can be determine as in the following equation;

$c_{i j}=\frac{1}{|N|} \sum_{k=1}^{|N|} d_{i}\left(x_{k}\right) * d_{j}\left(x_{k}\right) ; d_{i}\left(x_{k}\right)=t\left(x_{k}\right)-y_{i}\left(x_{k}\right) \forall i, j$

\section{Criteria for Evaluation of $O L C$}

Two qualitative performance measures have been used in this paper to describe the learning and generalization abilities of a given trained neural network. These are measured in terms of training error and generalization error. Training error is the MSE of the training data, while generalization error is the MSE of the testing data, which are computed as follows;

$T E_{j}=\frac{\sum_{k}\left(t\left(x_{k}\right)-y_{j}\left(x_{k}\right)\right)^{2}}{N_{1}} ; G E_{j}=\frac{\sum_{k}\left(t\left(x_{k}\right)-y_{j}\left(x_{k}\right)\right)^{2}}{N_{2}}$

It was initially [8] proposed that the optimal weight computation should based on the training error. To achive optimum performance of the network both the errors, $T E_{j}$ and $G E_{j}$ are equally important and have to be a minimum in a given situation. To achieve this, the error functions have been combined (10) and modified error function $O_{j}$ has been minimized thus:

$$
O_{j}=\frac{N_{1} \times T E_{j}+N_{2} \times G E_{j}}{N_{1}+N_{2}}
$$

Where, $N_{1}, N_{2}$ are the numbers of data set dedicated for training and testing with $N_{1}+N_{2}=N$. The data obtained from different loading cycles has been seen to exhibit a nearly Gaussian trend. 


\section{RESULTS AND Discussions}

The procedure for online estimation of HST and TOT described here was validated using the magnitudes of TOT rise and HST under a standard loading cycle pattern indicated earlier and also using loading pattern given in earlier published literature [2], [4], [9]. In all, seven datasets have been used for TOT modeling. The HST modeling has been illustrated using the four standard loading cycles as per the IEEE guide [3].

No guidelines seem to have been prescribed in the selection of optimum number of networks is to be combined. It has been reported elsewhere [10] that between 6 and 10 screened - network combination gives an acceptable accuracy. In the present work, eight networks have been trained using BR and combined optimally. The topologies of eight networks selected are different. Four of the networks have one hidden layer and other four have two hidden layers. This architecture is chosen to make the individual ANN perform efficiently in different subspaces. Further, this process of integration of all such networks is more efficient in the entire space.

In table II, 8-trained NNs and their MSE have been shown for a particular value of TOT. All the networks were trained for say, 200 iterations as has been done here. An error - duration

TABLE II

DETAILS OF EIGHT TRAINED NETWORKS

\begin{tabular}{|c|c|c|c|c|c|}
\hline \hline NN & Topology & No. of & \% Error & \multicolumn{2}{|c|}{ Optimal weights } \\
\cline { 5 - 6 } & & Epoch & MSE $\left(\mathrm{O}_{j}\right)$ & Uncon(M1) & Con(M2) \\
\hline \hline NN1 & $15-12-1$ & 200 & 0.4897 & -0.4701 & -0.4257 \\
NN2 & $15-13-2-1$ & 200 & 0.3318 & 0.2023 & 0.1913 \\
NN3 & $15-08-2-1$ & 200 & 0.2866 & 0.4512 & 0.4482 \\
NN4 & $15-04-1$ & 200 & 0.2639 & 0.4116 & 0.4065 \\
NN5 & $15-10-5-1$ & 200 & 0.2299 & 0.7053 & 0.7043 \\
NN6 & $15-10-1$ & 200 & 0.4218 & -0.5257 & -0.5289 \\
NN7 & $15-20-1$ & 200 & 0.4300 & 0.0064 & 0.1217 \\
NN8 & $15-15-2-1$ & 200 & 0.7791 & 0.2175 & 0.0827 \\
\hline \hline
\end{tabular}

curve in figure 2 , is plot of percentage error in TOT depicted as a function of the number of times the error is exceeded. A typical network from eight-trained networks has been designated as the best NN on the basis of mean square error (MSE). The MSE (see Eqn(10)) using OLCN are 0.119 and 0.123 for Constrained and unconstrained method respectively and are better than individual network, as can be seen from Table II. The magnitude of weights assigned to the eight-trained network has been included there. It may be observed that the OLCN using the method of constraints gives a better performance for the case shown here. The Error in estimation of TOT and HST have been shown in Table III. The MSE and maximum errors have been indicated for several cases. It can be seen that the constrained method of optimal weight outperforms the unconstrained method most of the time. It may also be seen that the performance shown here is, more often than not, better than the results in published work [2], [5].

In conclusion, it may be said that theoretical framework presented here is applicable to the estimation of both TOT and HST under dynamic loading and dynamic ambient. The generalization capability of the ANN model has been shown to have been augmented, by almost two fold, due to the incorporation

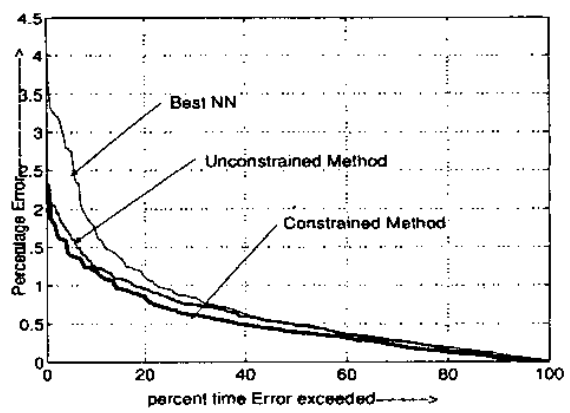

Fig. 2. Error Duration Curve

TABLE III

PERFORMANCE OF PROPOSED METHOD FOR COMPUTATION

\begin{tabular}{|c|c|c|c|c|c|c|}
\hline \multirow{3}{*}{$\begin{array}{c}\text { Load } \\
\text {-ing } \\
\text { type }\end{array}$} & \multicolumn{6}{|c|}{ Percentage(\%) Error $\left(\mathrm{O}_{j}\right)$} \\
\hline & \multicolumn{2}{|c|}{ Best NN } & \multicolumn{2}{|c|}{ OLC(MI) } & \multicolumn{2}{|c|}{ OLC NNs(M2) } \\
\hline & MSE & $\operatorname{Max}$ & MSE & $\operatorname{Max}$ & $\mathrm{MSE}$ & Max \\
\hline $\mathrm{NL}$ & 0.555 & 9.17 & 0.5405 & 8.10 & 0.1875 & 6.90 \\
\hline PLBN & 0.258 & 4.20 & 0.2313 & 3.85 & 0.1918 & 3.25 \\
\hline LTE & 0.145 & 4.20 & 0.1271 & 2.20 & 0.0939 & 2.30 \\
\hline STE & 0.295 & 4.20 & 0.2340 & 4.00 & 0.1443 & 1.95 \\
\hline [2] & 0.230 & 8.50 & 0.1227 & 5.25 & 0.1193 & 5.00 \\
\hline [4] & 2.295 & 9.00 & 0.7642 & 6.80 & 0.7726 & 7.10 \\
\hline [9] & 1.557 & 9.40 & 0.6396 & 6.50 & 0.4632 & 5.20 \\
\hline \multicolumn{7}{|c|}{ Simulation Results on HST data } \\
\hline NL & 0.14 & 3.75 & 0.0029 & 0.600 & 0.0007 & 0.300 \\
\hline PLBN & 0.47 & 4.27 & 0.073 & 2.048 & 0.0024 & 0.425 \\
\hline LTE & 6.70 & 8.00 & 1.231 & 3.900 & 0.2100 & 1.900 \\
\hline STE & 4.29 & 7.00 & 0.0877 & 2.400 & 0.1770 & 1.100 \\
\hline
\end{tabular}

of Bayesian Regularization and by the optimal combination of several networks. The accuracy of prediction and generalization capability is now claimed to be some what better than what is presented in the literature thus far. Also, the modelling time required is generally much shorter than in earlier cases.

\section{REFERENCES}

[1] M K Pradhan and T S Ramu "Estimation of the Hottest Spot Temperature (HST) in Power Transformers Considering Thermal Inhomogeniety of the Windings" IEEE Trans. Power Delivery (in press).

[2] B.C.Lesieutre, W.H.Hagman, J.L.Kirtley, "An improved tansfomer TOT modeling for use in an on line monitoring and diagnostic system", IEEE tran, on Power Delivery, vol.12 no.1, pp 249-256, Jan 1997.

[3] IEEE Guide for Loading Mineral-Oil-Immerssed Transformers IEEE std C57.91-1995, April 25, 1996.

[4] Qing He, Jennie Si, Daniel J.Tylavsky " Transformer Top Oil Modeling and Simulation", IEEE tran. Ind. App., vol.36 no.5, pp 1219-1225, Oct 2000.

[5] Qing He, Jennie Si, Daniel J.Tylavsky "Prediction TOT for Transformers using NN", IEEE Tran. PWRD, vol.15 no.4,pp 1205-1211, Oct 2000.

[6] S. Haykin "Neural Network-A comprehensive foundation", Second Edition, New Jersy, Prentice hall, 1999

[7] F.D. Foresee, M Hagan "Gauss-Newton Approximation to Bayesian Learning", IEEE Proc. of Conf. on ANN, Vol-4, pp.1930-1935, 1997

[8] Sherif Hashem Bruce Schmeiser "Improving Model Accuracy using Optimal Linear Combinations of Trained Neural Networks", IEEE Transactions on Neural Networks,no-6 pp.792-794 1992

[9] Daniel J.Tylavsky, Qing He. A McCulla, J R Hunt "Sources of Error in Substation Distribution Transformers Dynamic Modeling", IEEE tran, on Power Delivery, vol.15 no.1, pp 178-185, Jan 2000.

[10] V. Mani and S N Omkar 'Understanding Modeling Processes using Combination of trained neural networks", Taylor \& Francis: Int. jor. of production researchJuly 2002 , Vol-40, No-3, pp.547-559 